OPEN ACCESS

Edited by:

Enza Fazio,

University of Messina, Italy

Reviewed by:

Liu Kesong,

Beihang University, China

Emmanuel Stratakis,

Foundation for Research and

Technology Hellas, Greece

${ }^{*}$ Correspondence:

Feng Chen

chenfeng@mail.xjtu.edu.cn

Specialty section:

This article was submitted to Physical Chemistry and Chemical

Physics,

a section of the journal

Frontiers in Physics

Received: 15 May 2020

Accepted: 02 July 2020

Published: 12 August 2020

Citation:

Yong J, Yang Q, Hou X and Chen F (2020) Endowing Metal Surfaces With Underwater Superoleophobicity by

Femtosecond Laser Processing for

Oil-Water Separation Application.

Front. Phys. 8:305

doi: 10.3389/fphy.2020.00305

\section{Endowing Metal Surfaces With Underwater Superoleophobicity by Femtosecond Laser Processing for Oil-Water Separation Application}

\author{
Jiale Yong ${ }^{1}$, Qing Yang ${ }^{2}$, Xun Hou ${ }^{1}$ and Feng Chen ${ }^{1 *}$ \\ ${ }^{1}$ State Key Laboratory for Manufacturing System Engineering and Shaanxi Key Laboratory of Photonics Technology for \\ Information, School of Electronics \& Information Engineering, Xi'an Jiaotong University, Xi'an, China, ${ }^{2}$ School of Mechanical \\ Engineering, Xi'an Jiaotong University, Xi'an, China
}

In this paper, a widely applicable method that has promise in endowing various metals with oil-water separation ability by femtosecond laser processing is reported. We take an iron sheet as an example to show how to use a femtosecond laser to prepare underwater superoleophobic microstructure on metal substrates and then achieve oilwater separation. An array of through microholes was previously prepared on the iron sheet by a mechanical drilling process. Then, rough nanostructures were created on the surface of the porous sheet by femtosecond laser ablation, resulting in excellent superoleophobicity in water. When the mixture of water and oil was poured onto the porous underwater superoleophobic metal sheet, only the water in the mixture could pass through the sheet while the oil was intercepted, thus the oil/water mixture was successfully separated with high efficiency. Such a novel preparation process and separation manner can extend to different metal substrates. We believe that a wide range of metals like iron sheet can be potentially endowed with oil-water separation ability by femtosecond laser processing because femtosecond laser can process almost all of the metals.

Keywords: oil-water separation, femtosecond laser, metal surface, underwater superoleophobicity, porous structure

\section{INTRODUCTION}

Energy plays a vital role in our life, but the most used petrochemical energy also causes a series of environmental pollution problems. With the demand growth of global energy, oil spills occur frequently, and industrial oily wastewater is continuously discharged, causing not only serious ecological damage but also huge economic losses [1-7]. To deal with the pollution problems caused by the spilled oils and the industrial waste oils, various technologies and materials have been developed to achieve effective oil-water separation. Traditional methods for separating oilwater mixtures include adsorption, gravity separation, skimming, flotation, centrifugal separation $[2,5]$. Although these methods can separate most oil-water mixtures to some extent, they usually suffer from many obvious limitations, such as low separation efficiency, the need for external input driving energy, secondary pollution problems, etc. [2, 3, 5]. For instance, many porous materials such as foams, sponges, and textiles are usually used to alleviate pollution from the oil-leakage/spillage by absorbing oils from water. However, these materials absorb both oils and 
water, leading to a poor separation selectivity and a low efficiency. The collected oils are hard to be reused because of low purity. Also, such separation materials will be contaminated by oils just after one cycle of separation and then lose the absorbing ability. Secondary environmental pollution occurs with burning or burying the contaminated separation materials. These limitations force researchers to continuously develop more efficient and environmentally friendly separation materials and systems toward oil-water mixtures.

Based on the different interfacial behaviors between water and oils, porous materials with superwettability are applied in oil-water separation in recent years [1-5, 8-19]. The surfaces of such materials have superhydrophobicity/superoleophilicity or superoleophobicity/superhydrophilicity. Compared to the traditional oil-water separation materials, the superwetting materials show many advantages in separation performance. In 2004, Feng et al. prepared a superhydrophobic and superoleophilic (in the air) stainless steel mesh coated with polytetrafluoroethylene [8]. When the mixture of oil and water was poured onto the superwetting metal mesh, the water in the mixture was repelled by the mesh and remained above the mesh because of the superhydrophobicity. In contrast, the superoleophilicity allowed the oil to moisten and pass through the metal mesh. As a result, the oil-water mixture was successfully separated. In 2011, Xue et al. proposed a new strategy to achieve oil-water separation by using underwater superoleophobic porous membranes, such as hydrogel-coated stainless steel mesh [12]. The hydrogel-coated mesh exhibited superhydrophilicity in air but superoleophobicity immersed in water. As the oilwater mixture was poured onto the as-prepared mesh that was pre-wetted by water, only water could penetrate through the mesh, while oil was intercepted by the mesh, achieving oil-water separation. Following these separation principles, more and more superwetting porous materials have been prepared for oil-water separation [1-5]. However, the microfabrication methods to fabricate those superwetting materials are usually limited to specific substrate material; that is, a fabrication process just can make one or a handful of substrates become oil-water separation materials. For example, Zhang et al. prepared an underwater superoleophobic nanowire structure on the copper mesh surface based on a chemical-based oxidation method (immersing copper mesh in the solution of $\mathrm{NaOH}$ and $\left.\left(\mathrm{NH}_{4}\right)_{2} \mathrm{~S}_{2} \mathrm{O}_{8}\right)$ [20]. The structured mesh could separate both immiscible and dispersed oil/water mixtures. This chemical reaction can just endow copper with underwater superoleophobicity but would not be available to other metals. Metal is the most commonly used engineering material, and the category of metals is numerous. A widely applicable method that can endow various metals with superwettability and oil-water separation ability is still highly desired.

The features of ultra-short pulse width and extremely high peak power enable the femtosecond laser to theoretically process almost all of the solid materials [21-25]. In 2016, we reported that the thin polytetrafluoroethylene (PTFE) sheet exhibited durable ultralow-adhesive superhydrophobicity after femtosecond laser ablation [26]. Mechanical drilling process was also used to generate through microhole array on the rough superhydrophobic sheet. The as-prepared porous superhydrophobic sheet can separate various oil/water mixtures including oil and corrosive acid/alkali solutions. However, such superhydrophobic separation materials (named "oil-removing" materials) are easily blocked by the oil that adheres to the porous microstructure as it passes through, resulting in a great decrease in the separation efficiency. Although many laser-based oil/water separation materials have been developed, $[27,28]$ the fabrication of those materials by laser processing is not yet universal for the colorful category of metals.

In this paper, we take the iron sheet as an example to show how to use a femtosecond laser to prepare underwater superoleophobic microstructure on metal substrates and then achieve oil-water separation. Rough nanostructures were easily created on the iron surface by femtosecond laser processing, and the structured metal surface showed excellent underwater superoleophobicity. A porous underwater superoleophobic metal sheet was obtained by the combination of the mechanical drilling process and subsequent femtosecond laser ablation. The ability of the laser-induced porous underwater superoleophobic metal sheet to separate the water/oil mixture was demonstrated.

\section{METHODS}

\section{Femtosecond Laser Ablation}

A femtosecond laser (CoHerent, Libra-usp-1K-he-200) was used to create surface microstructure on a metal surface. The laser beam has a pulse duration of $50 \mathrm{fs}$, a center wavelength of $800 \mathrm{~nm}$, and a repetition of $1 \mathrm{kHz}$. The laser beam was focused onto the sample surface through an object lens (NA $=0.40$, Nikon, Japan). During laser processing, the manner of line-by-line scanning was adopted, which can refer to our previous works [29-31]. The laser power was set constant at $30 \mathrm{~mW}$ with the laser fluence of $7.46 \mathrm{~J}$ $\mathrm{cm}^{-2}$. The scanning speed and the scanning space were tuned by the control program.

\section{Fabrication of Superwetting Porous Metal Sheet}

The iron sheet (thickness of $0.3 \mathrm{~mm}$ ) was firstly drilled by a manmade mechanical system to form penetrating microholes that fully across the sheet. A mini drill bit with a diameter of $0.3 \mathrm{~mm}$ was controlled to pass through the metal sheet at the speed of $0.1 \mathrm{~mm} / \mathrm{s}$. Uniform open microholes array was obtained by the aid of a pre-designed program. Then, the surface of the porous sheet was further ablated by femtosecond laser to induce superwettability.

\section{Oil-Water Separation}

The laser-induced superwetting porous metal sheet was used as the separation membrane and was sandwiched between two glass tubes. Before separation, the sheet was pre-wetted by a small volume of water. Then, the mixture of oil (petroleum ether) and water with the volume rate of 1:1 was poured into the upper tube of the designed separation device. The separation system worked. For clear observation, the used water was dyed by methylene blue and showed blue color, while oil was dyed by Oil Red $\mathrm{O}$ and showed a red color. 


\section{Characterization}

The surface microstructure of the samples was observed by a scanning electron microscope (Gemini SEM 500, Zeiee, Germany) and a laser confocal microscope (LEXT-OLS4000, Olympus, Japan). The wettability of the as-prepared substrate was investigated by contact angle measurement (JC2000D, Powereach, China). Deionized water and 1,2-dichloroethane were adopted as the main tested liquids for water and oil, respectively.

\section{RESULTS AND DISCUSSION}

Nano-texture was easily induced on the iron surface by femtosecond laser processing. Figures $\mathbf{1 a}, \mathbf{b}, \mathbf{d}$ shows the scanning electronic microscopy (SEM) images of the iron surface after laser ablation. The sample was ablated at the scanning speed of $6 \mathrm{~mm} \mathrm{~s}^{-1}$ and the scanning space of $6 \mu \mathrm{m}$. The whole resultant surface is coated with a nano-ripples structure (Figures 1a,b). The width of every nano-ripple is $\sim 500 \mathrm{~nm}$. A large number of fine particles with the size of a few tens of nanometers further randomly distribute on the surface of nano-ripples (Figure 1d). Such a hierarchical nanostructure typically results from the interaction between femtosecond laser and metal substrates. The laser-induced hierarchical nanostructure is also verified by the three-dimensional and cross-sectional profiles by laser confocal scanning microscopy images (Figure 1c). The surface roughness of the laser-ablated iron substrate is measured to be $0.193 \mu \mathrm{m}$.

The wettability of the iron sample was investigated by measuring the contact angle (CA) and sliding angle (SA). A water droplet on the untreated flat iron surface has a water CA (WCA) of $78 \pm 3.9^{\circ}$, demonstrating that the iron surface is inherently hydrophilic (Figure 2A). The flat surface exhibits ordinary oleophobicity with an oil CA (OCA) of $107.8 \pm 2.8^{\circ}$ to oil droplet in water (Figure 2C). After femtosecond laser treatment, both the in-air hydrophilicity and the underwater oleophobicity of the iron substrate are enhanced by the laser-induced surface nanostructure. When a small water droplet was dripped on the laser-structured surface, it would spread out after touching the sample surface (Figure 2E). A small WCA of $12.8 \pm 2.4^{\circ}$ indicates that the iron surface shows quasi-superhydrophilicity after laser ablation (Figure 2B). Interestingly, the oil droplet can maintain a spherical shape with the OCA of $164 \pm 0.3^{\circ}$ on the rough surface in a water medium (Figure 2D). Therefore, the laser-ablated iron surface has underwater superoleophobicity. As an underwater oil droplet was moved to touch and then leave away the laser-structured surface, no shape deformation and residual of the oil droplet could be observed (Figure 2F). Once the sample was slightly tilted, the oil droplet could easily roll off the textured surface with the oil SA (OSA) of $\sim 1^{\circ}$ (Figure 2G). Apart from heavy oils, the droplets of light oils (e.g., petroleum ether) are also able to roll away on the underwater
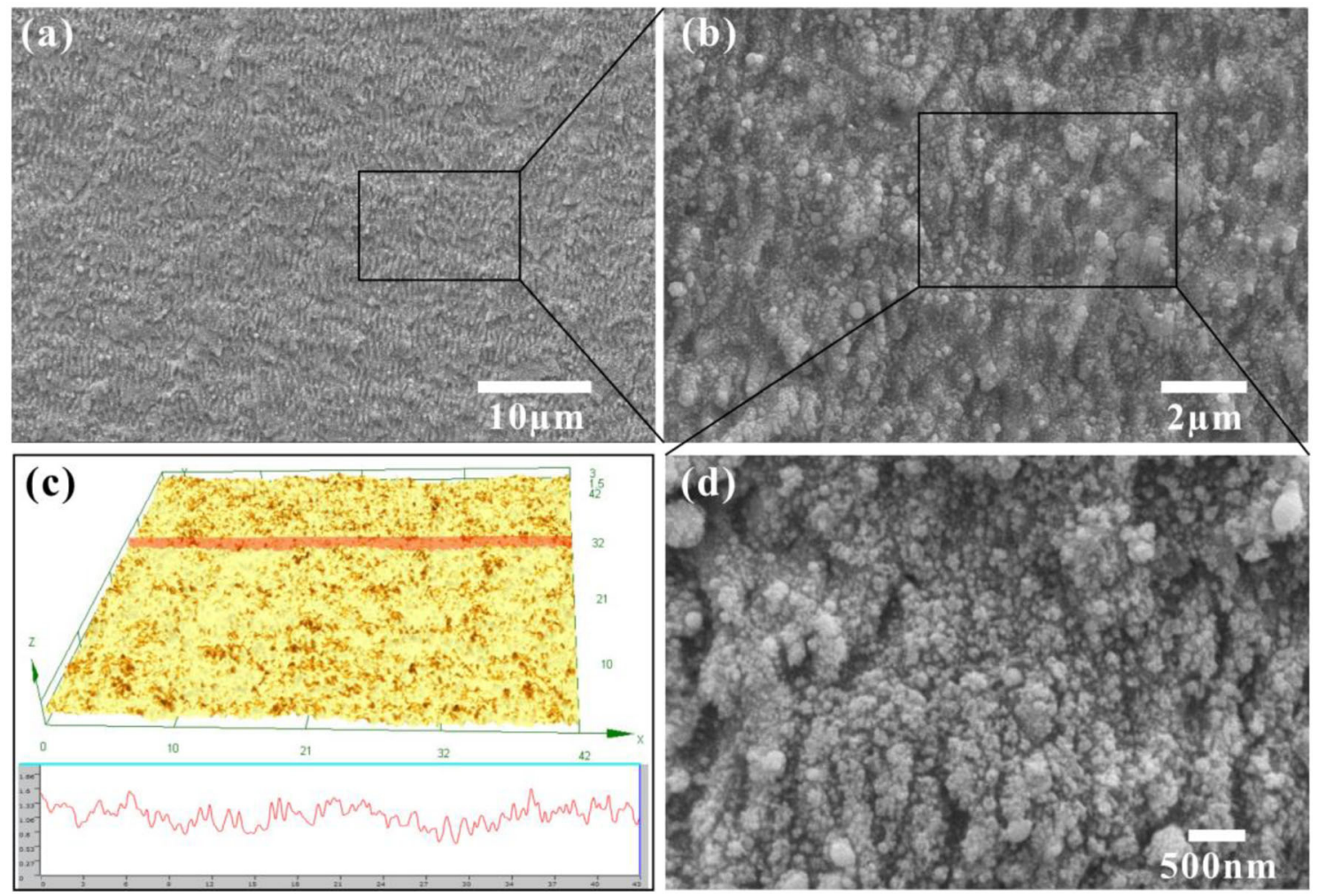

FIGURE 1 | Morphology of the iron surface after femtosecond laser ablation. (a,b,d) SEM images of the laser-ablated surface. (c) Laser confocal microscopy image of the laser-ablated surface. 

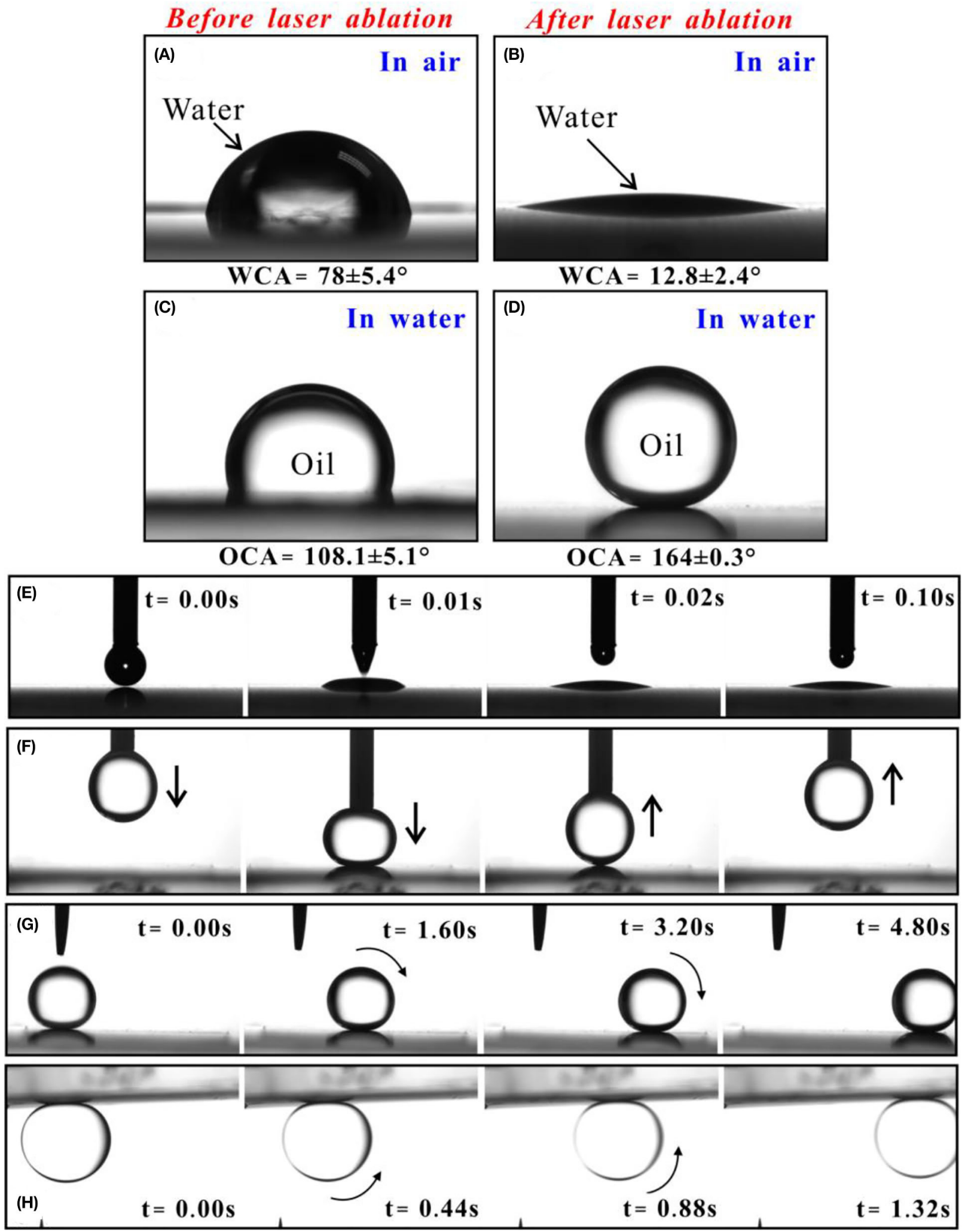

FIGURE 2 | Wettability of liquid droplet on the iron surface. (A) Water droplet on the untreated surfaces in air. (B) Water droplet on the laser-ablated surfaces in air. (C) Oil droplet on the untreated surfaces in water. (D) Oil droplet on the laser-ablated surfaces in water. (E) Dripping a small water droplet onto the laser-ablated surface in air. (F) Moving an underwater oil droplet to touch and then leave away the laser-structured surface. (G,H) Process of a droplet of (G) heavy oil and (H) light oil rolling on the laser-ablated surface in water. 


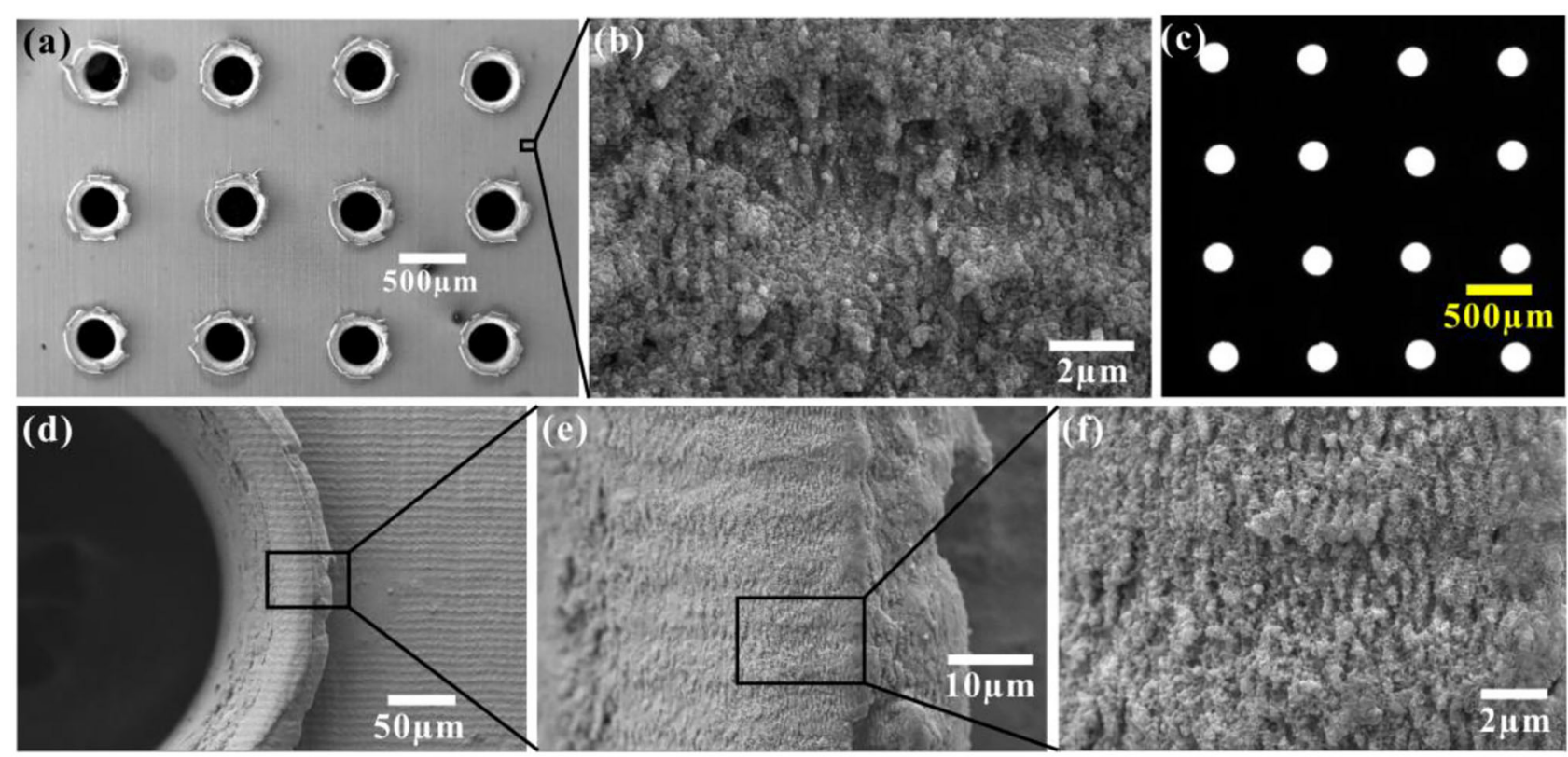

FIGURE 3 | Femtosecond laser-induced porous underwater superoleophobic metal sheet. (a) SEM image of the nanostructured porous sheet. (b) High-magnification SEM image of the domain between open microholes. (c) Optical microscopy photo of the porous sheet. The sample was lighted by white light from the backside. (d-f) SEM images of the edge ridge of a microhole.

superpolymphobic surface (Figure $\mathbf{2} \mathbf{H}$ ). The results indicate that the adhesion of the superoleophobic surface to various oil droplets in water is extremely low.

Femtosecond laser-induced surface microstructures play an important role in achieving underwater superoleophobicity on metal surfaces [32-34]. Generally, the rough surface microstructure can amplify the natural wettability of a solid substrate. Most of the metal substrates are inherently hydrophilic. Laser processing enables hierarchical micro/nanostructure to form on the surfaces of metal substrates, increasing the actual surface area of the substrates greatly. As a result, the hydrophilicity of the iron substrates is enhanced to quasisuperhydrophilicity by the laser-induced surface nanostructure. Water can completely wet the rough nanostructure of the iron surface because of the increased surface area and capillary action. The contact between water and the laser-induced nanostructure is at the Wenzel state [32]. Immersion in water makes all the nanostructure of the sample surface be wetted and occupied by water. When an oil droplet is further put on the metal surface, the oil will be repelled by the water trapped in the surface nanostructure due to the repulsive interaction between polar and non-polar molecules. The oil droplet is unable to penetrate the surface nanostructures of the metal substrate and can only touch the peak portion of the laser-induced nanostructures. The underwater oil droplet sits on a composite (water/solid) interface and is at the Cassie wetting state, [32] resulting in a solid/water/oil three-phase system. The hierarchical microstructure and the trapped water layer can effectively reduce the contact area between the oil droplet and the metal surface so that the adhesive force and adhesion of the laser-structured surface to underwater oils are very small. As a result, the metal surface presents underwater superpolymphobicity after femtosecond laser treatment.

As the scanning speed increases from 4 to $18 \mathrm{~mm} \mathrm{~s}^{-1}$ and the scanning space increases from 4 to $18 \mu \mathrm{m}$, the OCA value of an underwater oil droplet on the resultant iron surface remains above $150^{\circ}$ and the OSA value is always $<10^{\circ}$. Further increasing the laser scanning speed and scanning space leads to a decrease of OCA and an increase of OSA, resulting in a weak oleophobicity in water. The decline of oleophobicity is ascribed to the weak of the laser-induced surface rough microstructure. With increasing the laser scanning speed and scanning space, the focused laser pulses per area decline, weakening the ablation process and decreasing the roughness. In general, the femtosecond laser-induced superhydrophilic metal microstructures tend to gradually lose superhydrophilicity as well as underwater superoleophobicity storing in air because of absorbing environmental carbon contaminants [35]. Fortunately, we find that the storage of the laser-induced rough metal surfaces in water can maintain their superhydrophilicity and underwater superoleophobicity for a very long time. Therefore, low-adhesive underwater superoleophobicity can be achieved in a wide technology parameter range and is very stable in water.

A porous underwater superoleophobic sheet was easily fabricated by the combination of the mechanical drilling process and subsequent femtosecond laser ablation. As shown in Figure 3a, an array of microholes was formed on the resultant thin iron sheet. Observing with optical microscopy, backlight could directly pass through the laser-drilled microholes, demonstrating that the microholes are open and entirely 

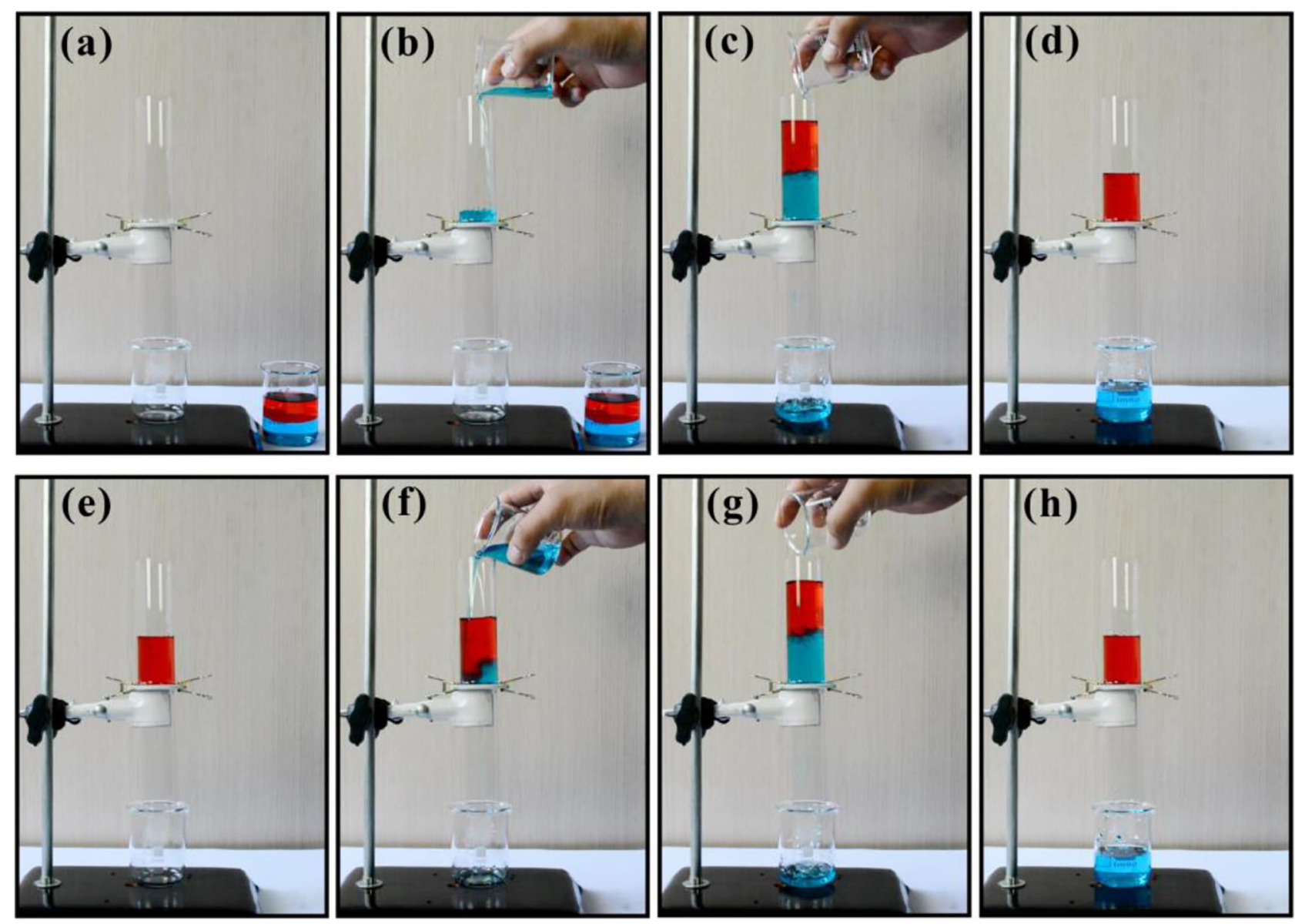

FIGURE 4 | Oil-water separation based on the femtosecond laser-induced porous underwater superoleophobic metal sheet. (a) Designed device before separation. (b) Pre-wetting the metal sheet with a little water. (c) Pouring the mixture of water (blue color) and oil (red color) into the separation device. (d) After separation. (e-h) Reactivating the separation process by adding new water into the device that just finished a cycle of oil-water separation.

through one side to another side of the sheet (Figure 3c). The diameter of the holes is about $300 \mu \mathrm{m}$, which is consistent with the diameter of the drill bit. The rest part between the microholes is fully covered by rough nano-ripples structure caused by femtosecond laser ablation (Figure 3b). Besides, the edge ridge of every microhole is also characterized by the nano-ripples (Figures 3d-f). Since the laser-induced nanostructures on the iron surface are superoleophobic in water, the resultant porous metal sheet has underwater superoleophobicity.

The features of underwater superoleophobicity and through microholes enable the porous metal sheet to be used as the core membrane for oil-water separation. As shown in Figure 4a, the porous underwater superoleophobic sheet was sandwiched between two glass tubes. Then, a small volume of water was dripped on the sheet to previously wet the metal sheet (Figure 4b). When the mixture of water (blue color) and oil (red color) was added into the designed separation device (Figure 4c), only water in the mixture completely permeated through the metal sheet, while oil was intercepted and stayed in the upper tube because of the underwater superoleophobicity of the laserinduced nanostructures (Figure 4d), so the oil-water mixture was successfully speared. The separation process was driven by just gravity, without any external forces. Also, the separation process can be repeated in many cycles. When new water was poured into the device that just finished a cycle of oil-water separation, the separation process would be reactivated (Figures $4 \mathbf{e}-\mathbf{h}$ ). The newly introduced water could pass through the metal sheet but oil was intercepted. The separation efficiency was calculated by $\eta=m_{1} / m_{0}$, where $m_{1}$ and $m_{0}$ are the mass of the collected oil and the oil before separation [36]. The measured separation efficiency reaches up to $97.8 \%$. Therefore, the femtosecond laserinduced porous underwater superoleophobic metal sheet exhibits high efficiency in oil-water separation.

The surprising separation ability of the resultant porous metal sheet is mainly caused by its remarkable underwater superoleophobicity. Underwater superoleophobicity is usually achieved on hydrophilic substrates. Interestingly, most kinds of metals are inherently hydrophilic. The femtosecond laser can ablate almost all of the known materials and then can create 
micro/nanoscale structures on the surface of various metals; [21-25] thereby underwater superoleophobicity can be easily achieved on different metals by femtosecond laser treatment, such as $\mathrm{Ti}$, [37] stainless steel, [38] $\mathrm{Al}$, [34] Cu, [39] Mo, and so on $[32,40]$. With the laser-induced underwater superoleophobic surface microstructures, a wide range of metallic sheets can potentially be endowed with the ability of oil-water separation.

\section{CONCLUSIONS}

In conclusion, we present a promising method to endow metal sheets with the ability of oil-water separation by femtosecond laser processing. Taking the iron sheet as an example, rough nanostructures were easily created on the iron surface after femtosecond laser ablation. Oil droplets on the laser-structured surface had an OCA of $164 \pm 0.3^{\circ}$ and an OSA of $\sim 1^{\circ}$ in water, revealing excellent underwater superoleophobicity of the laser-induced surface nanostructures. The porous underwater superoleophobic metal sheet was fabricated by the combination of the mechanical drilling process and subsequent femtosecond laser ablation. By using the laser-induced porous underwater superoleophobic sheet as the separation membrane, the mixture of water and oil was successfully separated with high efficiency (97.8\%). Because femtosecond laser can process almost all of the metals and endow various metals with underwater superoleophobicity, such a method of fabricating superwetting separation materials can extend to a wide range of metals substrates. Therefore, femtosecond laser processing is a widely

\section{REFERENCES}

1. Xue Z, Cao Y, Liu N, Feng L, Jiang L. Special wettable materials for oil/water separation. J Mater Chem A. (2014) 2:2445-60. doi: 10.1039/C3TA13397D

2. Wang B, Liang W, Guo Z, Liu W. Biomimetic super-lyophobic and superlyophilic materials applied for oil/water separation: a new strategy beyond nature. Chem Soc Rev. (2015) 44:336-61. doi: 10.1039/C4CS00220B

3. Gupta RK, Dunderdale GJ, England MW, Hozumi AJ. Oil/water separation techniques: a review of recent progresses and future directions. Mater Chem A. (2017) 5:16025-58. doi: 10.1039/C7TA02070H

4. Chu Z, Feng Y, Seeger S. Oil/water separation with selective superantiwetting/superwetting surface materials. Angew Chem Int Ed. (2015) 54:2328-38. doi: 10.1002/anie.201405785

5. Yong J, Huo J, Chen F, Yang Q, Hou X. Oil/water separation based on natural materials with super-wettability: recent advances. Phys Chem Chem Phys. (2018) 20:25140-63. doi: 10.1039/C8CP04009E

6. Li W, Yong J, Yang Q, Chen F, Fang Y, Hou X. Oil-water separation based on the materials with special wettability. Acta Phys Chem Sin. (2018) 34:45675. doi: 10.3866/PKU.WHXB201709211

7. Yong J, Chen F, Yang Q, Du G, Shan C, Huo J, et al. Oil-water separation: a gift from the desert. Adv Mater Interfaces. (2016). 3:1500650. doi: 10.1002/admi.201500650

8. Feng L, Zhang Z, Mai Z, Ma Y, Liu B, Jiang L, et al. A super-hydrophobic and super-oleophilic coating mesh film for the separation of oil and water. Angew Chem Int Ed. (2004) 43:2012-4. doi: 10.1002/anie.200353381

9. Kong LH, Chen XH, Yu LG, Wu ZS, Zhang PY. Superhydrophobic cuprous oxide nanostructures on phosphor-copper meshes and their oilwater separation and oil spill cleanup. ACS Appl Mater Interfaces. (2015) 7:2616-25. doi: 10.1021/am507620s

10. Li J, Kang R, Tang X, She H, Yang Y, Zha F. Superhydrophobic meshes that can repel hot water and strong corrosive liquids used for efficient gravity-driven oil/water separation. Nanoscale. (2016) 8:7638-48. doi: 10.1039/C6NR01298A applicable method that holds promise in endowing various metals with the ability of oil-water separation. We believe that the femtosecond laser-induced metallic separation sheets will have important applications in practically solving the pollution problems caused by oil spills and industrial oily wastewater.

\section{DATA AVAILABILITY STATEMENT}

The original contributions presented in the study are included in the article/supplementary material, further inquiries can be directed to the corresponding author/s.

\section{AUTHOR CONTRIBUTIONS}

JY designed the experiments and wrote the manuscript. FC directed and supervised the research. QY and XH contributed toward significant discussions and revised the paper. All authors contributed to the article and approved the submitted version.

\section{FUNDING}

This work was supported by the National Key Research and Development Program of China under the Grant no. 2017YFB1104700, the National Science Foundation of China under the Grant nos. 61805192 and 61875158, the International Joint Research Laboratory for Micro/Nano Manufacturing and Measurement Technologies, and the Fundamental Research Funds for the Central Universities.

11. Xue CH, Li YR, Hou YL, Zheng L, Ma JZ. Self-roughened superhydrophobic coatings for continuous oil-water separation. J Mater Chem A. (2015) 3:10248-53. doi: 10.1039/C5TA01014D

12. Xue Z, Wang S, Lin L, Chen L, Liu M, Feng L, et al. A novel superhydrophilic and underwater superoleophobic hydrogel-coated mesh for oil/water separation. Adv Mater. (2011) 23:4270-3. doi: 10.1002/adma.2011 02616

13. Liu YQ, Zhang YL, Fu XY, Sun HB. Bioinspired underwater superoleophobic membrane based on a graphene oxide coated wire mesh for efficient oil/water separation. ACS Appl Mater Interfaces. (2015) 7:20930-6. doi: 10.1021/acsami.5b06326

14. Zhang E, Cheng Z, Lv T, Qian Y, Liu Y. Anti-corrosive hierarchical structured copper mesh film with superhydrophilicity underwater low adhesive superoleophobicity for highly efficient oil-water separation. J Mater Chem A. (2015) 3:13411-7. doi: 10.1039/C5TA02053K

15. Tao M, Xue L, Liu F, Jiang L. An intelligent superwetting PVDF membrane showing switchable transport performance for oil/water separation. Adv. Mater. (2014) 26:2943-8. doi: 10.1002/adma.2013 05112

16. Li G, Fan H, Ren F, Zhou C, Zhang Z, Xu B, et al. Multifunctional ultrathin aluminum foil: oil/water separation and particle filtration. J Mater Chem A. (2016) 4:18832-40. doi: 10.1039/C6TA08231A

17. Zhu Q, Pan Q. Mussel-inspired direct immobilization of nanoparticles and application for oil-water separation. ACS Nano. (2014) 8:14029. doi: $10.1021 / \mathrm{nn} 4052277$

18. Cao C, Ge M, Huang J, Li S, Deng S, Zhang S, et al. Robust fluorinefree superhydrophobic PDMS-Ormosil@Fabrics for highly effective selfcleaning efficient oil-water separation. J Mater Chem A. (2016) 4:1217987. doi: 10.1039/C6TA04420D

19. Su L, Wang H, Niu M, Fan X, Ma M, Shi Z. Ultralight, recoverable, and high-temperature-resistant SiC nanowire aerogel. ACS Nano. (2018) 12:310311. doi: $10.1021 /$ acsnano.7b08577 
20. Zhang F, Zhang WB, Shi Z, Wang D, Jin J, Jiang L. Nanowire-haired inorganic membranes with superhydrophilicity and underwater ultralow adhesive superoleophobicity for high-efficiency oil/water separation. Adv Mater. (2013) 25:4192-8. doi: 10.1002/adma.201301480

21. Vorobyev AY, Guo C. Direct femtosecond laser surface nano/microstructuring and its applications. Laser Photon Rev. (2013) 7:385. doi: 10.1002/lpor.201200017

22. Sugioka K, Cheng Y. Femtosecond laser three-dimensional micro- and nanofabrication. Appl Phys Rev. (2014) 1:041303. doi: 10.1063/1.4904320

23. Sugioka K, Cheng Y. Ultrafast lasers-reliable tools for advanced materials processing. Light Sci Appl. (2014) 3:e149. doi: 10.1038/lsa.2014.30

24. Bai X, Yang Q, Fang Y, Zhang J, Yong J, Hou X, et al. Superhydrophobicitymemory surfaces prepared by a femtosecond laser. Chem Eng J. (2020) 383:123143. doi: 10.1016/j.cej.2019.123143

25. Yong J, Zhang C, Bai X, Zhang J, Yang Q, Hou X, et al. Designing "supermetalphobic" surfaces that greatly repel liquid metal by femtosecond laser processing: does the surface chemistry or microstructure play a crucial role? Adv Mater Interfaces. (2020) 7:1901931. doi: 10.1002/admi.2019 01931

26. Yong J, Fang Y, Chen F, Huo J, Yang Q, Bian H, et al. Femtosecond laser ablated durable superhydrophobic PTFE films with micro-through-holes for oil/water separation: separating oil from water and corrosive solutions. Appl Surf Sci. (2016) 389:1148-55. doi: 10.1016/j.apsusc.2016.07.075

27. Yong J, Yang Q, Guo C, Chen F, Hou X. A review of femtosecond laser-structured superhydrophobic or underwater superoleophobic porous surfaces/materials for efficient oil/water separation. RSC Adv. (2019) 9:1247095. doi: 10.1039/C8RA10673H

28. Alnaser AS, Khan SA, Ganeev RA, Stratakis E. Recent advances in femtosecond laser-induced surface structuring for oil-water separation. Appl Sci. (2019) 9:1554. doi: 10.3390/app9081554

29. Yong J, Chen F, Yang Q, Zhang D, Du G, Si J, et al. Femtosecond laser weaving superhydrophobic patterned PDMS surfaces with tunable adhesion. J Phys Chem C. (2013) 117:24907-12. doi: 10.1021/jp408863u

30. Yong J, Singh SC, Zhan Z, Mohamed E, Chen F, Guo C. Femtosecond laser-produced underwater "superpolymphobic" nanorippled surfaces: repelling liquid polymers in water for application of controlling polymer shape and adhesion. ACS Appl Nano Mater. (2019) 2:7362-71. doi: 10.1021/acsanm.9b01869

31. Yong J, Yang Q, Chen F, Zhang D, Farooq U, Du G, et al. A simple way to achieve superhydrophobicity, controllable water adhesion, anisotropic sliding, and anisotropic wetting based on femtosecondlaser-induced line-patterned surfaces. J Mater Chem A. (2014) 2:5499507. doi: 10.1039/C3TA14711H

32. Yong J, Chen F, Yang Q, Huo J, Hou X. Superoleophobic surfaces. Chem Soc Rev. (2017) 46:4168-217. doi: 10.1039/C6CS00751A
33. Yong J, Chen F, Li M, Yang Q, Fang Y, Huo J, et al. Remarkably simple achievement of superhydrophobicity, superhydrophilicity, underwater superoleophobicity, underwater superoleophilicity, underwater superaerophobicity, and underwater superaerophilicity on femtosecond laser ablated PDMS surfaces. J Mater Chem A. (2017) 5:25249-57. doi: 10.1039/C7TA07528F

34. Yong J, Singh SC, Zhan Z, Chen F, Guo C. How to obtain six different superwettabilities on a same microstructured pattern: relationship between various superwettabilities in different solid/liquid/gas systems. Langmuir. (2019) 35:921-7. doi: 10.1021/acs.langmuir.8b03726

35. Yan H, Rashid MRBA, Khew SY, Li F, Hong M. Wettability transition of laser textured brass surfaces inside different mediums. Appl Surf Sci. (2018) 427:369-75. doi: 10.1016/j.apsusc.2017.08.218

36. Li J, Li D, Yang Y, Li J, Zha F, Lei Z. A prewetting induced underwater superoleophobic or underoil (super) hydrophobic waste potato residuecoated mesh for selective efficient oil/water separation. Green Chem. (2016) 18:541-9. doi: 10.1039/C5GC01818H

37. Yong J, Chen F, Yang Q, Zhang D, Farooq U, Du G, et al. Bioinspired underwater superoleophobic surface with ultralow oil-adhesion achieved by femtosecond laser microfabrication. J Mater Chem A. (2014) 2:87905. doi: 10.1039/C4TA01277A

38. Yong J, Singh SC, Zhan Z, Huo J, Chen F, Guo C. Reducing adhesion for dispensing tiny water/oil droplet and gas bubble by femtosecond laser-treated needle nozzles: superhydrophobicity, superoleophobicity, and superaerophobicity. ChemNanoMat. (2019) 5:241-9. doi: 10.1002/cnma.201800495

39. Zhang J, Chen F, Yang Q, Yong J, Huo J, Fang Y, et al. A widely applicable method to fabricate underwater superoleophobic surfaces with low oiladhesion on different metals by a femtosecond laser. Appl Phys A. (2017) 123:594. doi: 10.1007/s00339-017-1195-8

40. Yong J, Chen F, Yang Q, Jiang Z, Hou X. A review of femtosecond-laserinduced underwater superoleophobic surfaces. Adv Mater Interfaces. (2018) 5:1701370. doi: 10.1002/admi.201701370

Conflict of Interest: The authors declare that the research was conducted in the absence of any commercial or financial relationships that could be construed as a potential conflict of interest.

Copyright (c) 2020 Yong, Yang, Hou and Chen. This is an open-access article distributed under the terms of the Creative Commons Attribution License (CC BY). The use, distribution or reproduction in other forums is permitted, provided the original author(s) and the copyright owner(s) are credited and that the original publication in this journal is cited, in accordance with accepted academic practice. No use, distribution or reproduction is permitted which does not comply with these terms. 